\title{
The role of lipoic acid in product formation by Enterococcus faecalis NCTC 775 and reconstitution in vivo and in vitro of the pyruvate dehydrogenase complex
}

\author{
Jacky L. Snoep, Mark van Bommel, Frank Lubbers, M. Joost Teixeira de Mattos* and \\ OENSE M. NEIJSSEL
}

Department of Microbiology, Biotechnology Centre, University of Amsterdam, Nieuwe Achtergracht 127, 1018 WS Amsterdam, The Netherlands

(Received II November 1992; revised 18 January 1993; accepted 9 February 1993)

\begin{abstract}
The role of the pyruvate dehydrogenase complex (PDC) in the formation of different fermentation products by Enterococcus faecalis was studied. This organism was grown on a semi-defined medium under various conditions in the presence or absence of lipoic acid, an essential cofactor of the enzyme complex. When grown on a medium without added lipoic acid, a very low activity, both in vivo and in vitro, of the PDC was observed. When pyruvate served as the energy source, lipoic acid was found to be essential for growth under anaerobic conditions at low culture $\mathrm{pH}$ values. The presence of lipoic acid in the culture medium had a marked effect on the production of acetoin: in the presence of lipoic acid, acetoin was produced only when the intracellular pyruvate concentration was relatively high, whereas in the absence of lipoic acid, acetoin was a common product. Under potassium-limited conditions, lactate was the main product and culture $\mathrm{pH}$ significantly affected the bacterial dry weight. After instantaneous addition of lipoic acid to a glucose + pyruvate-limited chemostat culture, an immediate activation of the PDC took place as deduced from the change in fermentation pattern. Reconstitution of the PDC by the addition of lipoic acid was also possible in cell-free extracts, although pre-incubation with ATP and lipoic acid for 90 min was necessary for maximal activation. The effects of an active PDC on product formation and the physiological role of the complex under anaerobic growth conditions are discussed.
\end{abstract}

\section{Introduction}

The pyruvate dehydrogenase complex (PDC) occupies a key position between glycolysis and the citric acid cycle. The properties of the PDC have been extensively investigated in Escherichia coli (for reviews see Reed, 1974; Wieland, 1983; Perham et al., 1987; Yeaman, 1989; Patel \& Roche, 1990; Perham, 1991). It is generally assumed that the synthesis and activity of the PDC is dependent upon the presence of oxygen and that it is completely inactive under anaerobic conditions. Surprisingly, in Enterococcus faecalis the PDC was found to be active under anaerobic growth conditions (Snoep et al., 1990). This could be attributed to the low sensitivity of

\footnotetext{
${ }^{*}$ Author for correspondence. Tel. 20 5257066; fax 205255698.
}

Abbreviations: $\alpha$-ALS, $\alpha$-acetolactate synthase; LDH, lactate dehydrogenase; PDC, pyruvate dehydrogenase complex; PFL, pyruvate formate-lyase; SDM, semi-defined medium. this PDC for NADH and its high level of expression even under anaerobic conditions (Snoep et al., 1992a).

Besides the PDC complex, three other enzymes are known to catabolize pyruvate in E. faecalis: pyruvate formate-lyase (PFL), lactate dehydrogenase (LDH) and $\alpha$-acetolactate synthase ( $\alpha$-ALS). The simultaneous presence of these enzymes raises the question as to how the catabolic flux through these enzymes is regulated. The distribution of the carbon flux over the PDC and PFL was observed to be correlated to the redox potential of the NAD/NADH couple (Snoep et al., 1991). LDH is known to be stimulated by fructose-1,6-bisphosphate (Wolin, 1964; Wittenberger \& Angelo, 1970), a glycolytic intermediate that is present in high amounts during growth under conditions of glucose excess (Yamada \& Carlsson, 1975). Previous experiments showed that $\alpha$ ALS has a very low affinity for pyruvate $\left[K_{\mathrm{m}}=50 \mathrm{~mm}\right.$ (Snoep et al., 1992b)], which implies that this enzyme can be active only when the intracellular pyruvate concentration is high. In contrast, the affinity of the 
other three enzymes for pyruvate is much higher $\left[K_{\mathrm{m}}\right.$ values of 1-5 mM (Takahashi et al., 1982; Wittenberger \& Angelo, 1970; Snoep et al., 1992a)].

An intriguing question is how the catabolic fluxes will be altered by the absence or activation of one of these enzymes. We have used the inability of $E$. faecalis to synthesize lipoic acid, an essential cofactor of the PDC, to study the effect of PDC on catabolism of the energy source and product formation. In addition we report on the kinetics of the reconstitution in chemostat culture and in cell-free extracts of the apo-PDC (PDC without lipoamide).

\section{Methods}

Micro-organism and growth conditions. Enterococcus faecalis NCTC 775 was maintained on beads in a reinforced clostridial medium (Oxoid) with $50 \%(\mathrm{w} / \mathrm{v})$ glycerol at $-20^{\circ} \mathrm{C}$. Organisms were cultured in a $700 \mathrm{ml}$ Modular Fermentor Series III (L. H. Engineering) as described previously (Snoep et al., 1990). They were grown on a semidefined medium (SDM), containing simple salts as specified by Evans et al. (1970), but with nitrilotriacetic acid $\left(0.38 \mathrm{~g} \mathrm{l}^{-1}\right)$ rather than citrate as a chelating agent, and with the following additions $\left(1^{-1}\right)$ : Tween- 80 $(0.2 \mathrm{~g})$, vitamin assay casaminoacids $(4.0 \mathrm{~g}$, Difco), L-asparagine $(0.3 \mathrm{~g})$, L-glutamine $(0.3 \mathrm{~g})$, L-cysteine $(0.1 \mathrm{~g})$, L-tryptophan $(0.05 \mathrm{~g})$, guanine $(5 \mathrm{mg})$, adenine $(5 \mathrm{mg})$, xanthine $(5 \mathrm{mg})$, uracil $(5 \mathrm{mg})$, nicotinic acid $(1 \mathrm{mg})$, inosine (1 mg), riboflavin (1 mg), thiamin (1 mg), pantothenate $(1 \mathrm{mg})$, pyridoxine $(1 \mathrm{mg})$, pyridoxal $(1 \mathrm{mg})$, orotic acid $(1 \mathrm{mg})$, biotin $(0.1 \mathrm{mg}), p$-aminobenzoic acid $(0.1 \mathrm{mg})$, folic acid $(0.1 \mathrm{mg})$ and cyanocobalamin $(0.01 \mathrm{mg})$. Amino acids were sterilized at $110^{\circ} \mathrm{C}$, whereas the other compounds were dissolved either at high or at low $\mathrm{pH}$ (as required) and filter sterilized (Seitz filter plates, type EKS). If $\mathrm{D}, \mathrm{L}-\alpha$-lipoic acid was added to the medium, a standard concentrated solution (about $0.5 \mathrm{M}$ ) was made in ethanol. Limitation of energy source was achieved by adding, respectively, 50-100 mM-pyruvate or 25-50 mm-glucose to the medium. The same concentrations were used when cells were grown on mixed substrates. The pyruvate solution was adjusted to $\mathrm{pH} 3.5$ by the addition of $\mathrm{NaOH}$ and subsequently sterilized by filtration (Seitz filter plates, type EKS). Glucose was sterilized at $110^{\circ} \mathrm{C}$. Potassium-limited conditions were obtained by adding $1 \mathrm{~mm}-\mathrm{KCl}$ (final concentration) to the medium with the glucose concentration raised to $250 \mathrm{~mm}$.

Analyses. Steady state bacterial dry weight was measured by the procedure of Herbert et al. (1971). Pyruvate, lactate, formate, acetate and acetoin were determined by HPLC as described previously (Snoep et al., 1990). Carbon dioxide production and oxygen consumption by the cultures were determined by passing the effluent gas from the fermenter through an Infralyt carbon dioxide analyser (Junkalor) and an oxygen analyser (Taylor Servomex Type OA 272). Protein was assayed according to the biuret method (Gornall et al., 1949).

Pulse experiments. A sterilized solution of pyruvate was injected into the culture vessel of an anaerobic glucose + pyruvate-limited culture by means of a syringe ( $50 \mathrm{~mm}$ final concentration). Simultaneously the medium pump was switched off. Samples were taken at regular time intervals and cells and supernatant were separated by centrifugation in an Eppendorf centrifuge. Growth rates were calculated from the change in bacterial dry weight during the pulse.

Reconstitution experiments. Reconstitution in vitro was performed at room temperature in $50 \mathrm{~mm}$-sodium phosphate buffer, $\mathrm{pH} 7.0$, con-

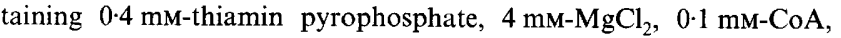

1.2 mM-dithiothreitol, $0.8 \mathrm{mM}-\mathrm{NAD}, 80 \mu \mathrm{M}-\mathrm{ATP}$, and $60 \mu \mathrm{M}-\mathrm{D}, \mathrm{L}-\alpha-$ lipoic acid and cell-free extract (approx. $1 \mathrm{mg}$ protein $\mathrm{ml}^{-1}$ final concentration). After various time intervals, the overall $\mathrm{PDC}$ activity was measured spectrophotometrically, by NADH formation, in the same mix. The reaction was started by the addition of sodium pyruvate (4 mM final concentration).

Reconstitution in vivo was performed by adding lipoic acid ( $10 \mu \mathrm{M}$ final concentration) to the culture vessel of the chemostat (see also Pulse experiments above). Enzyme activities in vivo were calculated from the specific product formation rates, i.e. $J_{\mathrm{PDC}}=$ $q_{\text {acetate }}+q_{\text {ethanol }}-q_{\text {formate }}$, as discussed previously (Snoep et al., 1990).

\section{Results}

E. faecalis was grown on SDM under glucose-, pyruvateand glucose + pyruvate-limited (energy-source-limited), and potassium-limited (glucose excess) conditions. Under all these conditions, steady state fluxes through the four pyruvate catabolizing enzymes were measured with special attention to the flux through the PDC $\left(J_{\mathrm{PDC}}\right)$ in the presence or absence of lipoic acid.

The fermentation pattern with glucose as sole energy source was of a mixed acid type, with higher lactate production rates at lower culture $\mathrm{pH}$ values (Table 1). Addition of lipoic acid to the medium $(4.9 \mu \mathrm{M}$ final concentration) resulted in an increase in $J_{\mathrm{PDC}}$ from 0.2 to $1.4 \mathrm{mmol}$ (g dry wt) $)^{-1} \mathrm{~h}^{-1}$ in cells grown at $\mathrm{pH} 6.0$. Concomitant with the increase in the $J_{\mathrm{PDC}}$, a decrease in the specific rate of formate production $\left(q_{\text {formate }}\right)$ and an increase in the $q_{\text {acetate }}$ and $q_{\text {ethanol }}$ was observed. In cells grown at $\mathrm{pH} \mathrm{7,} \mathrm{no} \mathrm{large} \mathrm{flux} \mathrm{through} \mathrm{the} \mathrm{PDC} \mathrm{was}$ observed, whether or not lipoic acid was added to the medium (Table 1).

The effect of the PDC on product formation is expected to be larger with higher turnover rates of pyruvate. Therefore, E. faecalis was grown under aerobic and anaerobic conditions on SDM with glucose +

Table 1. Specific rates $(q)$ of product formation and glucose consumption by E. faecalis grown in anaerobic chemostat culture under glucose-limited conditions on $\operatorname{SDM}\left(D=0 \cdot 1 h^{-1}, 37^{\circ} \mathrm{C}\right)$

The final concentration of lipoic acid added to the medium is shown in $\mu$ M. $q$ values are expressed as mmol $(\mathrm{g} \text { dry wt })^{-1} \mathrm{~h}^{-1}$. Glc, glucose; Ac, acetate; Lac, lactate; For, formate; EtOH, ethanol. Carbon recoveries and redox balances varied between 90 and $110 \%$. Values are the average of three independent chemostat runs, $\mathrm{SD}<5 \%$ of the mean.

\begin{tabular}{|c|c|c|c|c|c|c|c|}
\hline $\mathrm{pH}$ & $\begin{array}{c}\text { Lipoic } \\
\text { acid } \\
\text { concn }\end{array}$ & $q_{\mathrm{Glc}}$ & $q_{\mathrm{Ac}}$ & $q_{\mathrm{EtOH}}$ & $q_{\text {Lac }}$ & $q_{\mathrm{CO}_{2}}$ & $q_{\text {For }}$ \\
\hline 7.0 & 0 & $-4 \cdot 6$ & 1.5 & 1.6 & 4.5 & 0.5 & $3 \cdot 0$ \\
\hline $6 \cdot 0$ & 0 & $-5 \cdot 1$ & 1.0 & 1.3 & 6.6 & 0.5 & $2 \cdot 1$ \\
\hline $7 \cdot 0$ & 5 & $-3 \cdot 5$ & $1 \cdot 0$ & 1.3 & $4 \cdot 0$ & 0.3 & $2 \cdot 1$ \\
\hline $6 \cdot 0$ & 5 & $-6 \cdot 2$ & $1 \cdot 2$ & 1.9 & 8.7 & $1 \cdot 0$ & 1.7 \\
\hline
\end{tabular}


Table 2. Specific rates ( $q$ ) of product formation and of substrate consumption by E. faecalis grown anaerobically $\left(\mathrm{O}_{2},-\right)$ or aerobically $\left(\mathrm{O}_{2},+\right)$ in chemostat culture under glucose + pyruvate-limited conditions on $\operatorname{SDM}\left(D=0 \cdot 1 h^{-1}, \mathrm{pH} 6 \cdot 0,37^{\circ} \mathrm{C}\right)$

The final concentration of lipoic acid added to the medium is shown in $\mu \mathrm{M} . q$ values are expressed as $\mathrm{mmol}(\mathrm{g} \mathrm{dry} \mathrm{wt})^{-1} \mathrm{~h}^{-1}$. Abbreviations as for Table 1 except Acet, acetoin; Pyr, pyruvate. Carbon recoveries and redox balances varied between 90 and $110 \%$. Values are the average of three independent chemostat runs, $\mathrm{SD}<5 \%$ of the mean.

\begin{tabular}{|c|c|c|c|c|c|c|c|c|c|}
\hline $\begin{array}{l}\text { Lipoic } \\
\text { acid } \\
\text { concn }\end{array}$ & $\mathrm{O}_{2}$ & $q_{\mathrm{Glc}}$ & $q_{\mathrm{Pyr}}$ & $q_{\mathrm{Ac}}$ & $q_{\mathrm{Lac}}$ & $q_{\mathrm{O}_{2}}$ & $q_{\mathrm{CO}_{2}}$ & $q_{\text {For }}$ & $q_{\text {Acet }}$ \\
\hline 0 & - & -5.9 & $-4 \cdot 8$ & 3.8 & $11 \cdot 8$ & & 1.5 & 3.5 & 0.6 \\
\hline 0.2 & - & $-6 \cdot 0$ & $-4 \cdot 8$ & $2 \cdot 8$ & 11.9 & & $2 \cdot 3$ & $1 \cdot 0$ & 0.1 \\
\hline 0 & + & -5.9 & $-6 \cdot 0$ & 1.5 & 5.8 & -4.0 & 9.8 & 0 & $4 \cdot 1$ \\
\hline $2 \cdot 4$ & + & $-4 \cdot 2$ & $-4 \cdot 1$ & $6 \cdot 2$ & $4 \cdot 7$ & $-5 \cdot 4$ & $7 \cdot 2$ & 0 & 0.2 \\
\hline
\end{tabular}

Table 3. Typical values of the specific rates $(q)$ of product formation and of substrate consumption by $E$. faecalis grown in anaerobic chemostat culture under pyruvate-limited conditions on SDM $\left(D=0 \cdot 1 \mathrm{~h}^{-1}\right.$, $37^{\circ} \mathrm{C}$ )

The final concentration of lipoic acid added to the medium is shown in $\mu$ M. $q$ values are expressed as mmol $\left(\mathrm{gdry} \mathrm{wt}^{-1} \mathrm{~h}^{-1}\right.$. Abbreviations as for Tables 1 and 2. Acetoin was not formed at detectable concentrations under any of the conditions. Carbon recoveries and redox balances varied between 90 and $110 \%$. Values are the average of three determinations on samples of steady-state chemostat cultures.

\begin{tabular}{|c|c|c|c|c|c|c|}
\hline $\mathrm{pH}$ & $\begin{array}{l}\text { Lipoic } \\
\text { acid } \\
\text { concn }\end{array}$ & $q_{\text {Pуг }}$ & $q_{\mathrm{Ac}}$ & $q_{\text {Lac }}$ & $q_{\mathrm{CO}_{2}}$ & $q_{\text {For }}$ \\
\hline $7 \cdot 5$ & 0 & $-11 \cdot 2$ & $10 \cdot 5$ & 0 & 0.8 & $10 \cdot 2$ \\
\hline $7 \cdot 5$ & 5 & $-12 \cdot 6$ & $12 \cdot 1$ & 0 & 0.8 & $10 \cdot 9$ \\
\hline 6.0 & 5 & $-18 \cdot 8$ & $13 \cdot 5$ & $7 \cdot 2$ & $10 \cdot 5$ & $4 \cdot 1$ \\
\hline
\end{tabular}

Table 4. Typical values of the specific rates $(q)$ of product formation and of glucose consumption by $E$. faecalis grown in anaerobic chemostat culture under potassium-limited conditions on SDM $\left(D=0 \cdot 1 \mathrm{~h}^{-1}\right.$, $37^{\circ} \mathrm{C}$ )

No lipoic acid was added to the medium. $q$ values are expressed as mmol (g dry wt $)^{-1} h^{-1}$. Abbreviations as for Tables 1 and 2 . Redox balances varied between 90 and $110 \%$. Carbon balances varied between 80 and $85 \%$. Values are the average of three determinations on samples of steady-state chemostat cultures.

\begin{tabular}{ccccccc}
\hline \hline $\mathrm{pH}$ & $q_{\mathrm{Glc}}$ & $q_{\mathrm{Ac}}$ & $q_{\mathrm{EtOH}}$ & $q_{\mathrm{Lac}}$ & $q_{\mathrm{CO}_{2}}$ & $q_{\mathrm{For}}$ \\
\hline 8.0 & -12.5 & 2.0 & $2 \cdot 1$ & $16 \cdot 8$ & 0.3 & 4.9 \\
7.0 & $-12 \cdot 8$ & 1.3 & 1.4 & $18 \cdot 1$ & 0.3 & $2 \cdot 8$ \\
6.0 & -13.8 & 0.6 & 0.8 & 20.5 & 0.5 & 1.3 \\
\hline \hline
\end{tabular}

pyruvate as energy sources. No ethanol was formed and during anaerobic growth all NADH was oxidized via the reduction of pyruvate to lactate, resulting in a much higher $q_{\text {lactate }}$ as compared to growth with glucose as sole energy source (Table 2). Addition of lipoic acid to the medium resulted in an increase of $J_{\mathrm{PDC}}$ from 0.3 to $1.8 \mathrm{mmol}(\mathrm{g} \text { dry wt })^{-1} \mathrm{~h}^{-1}$. Even more significant was the effect of lipoic acid addition under aerobic conditions. PFL is extremely oxygen sensitive (Takahashi et al., 1982) and in the presence of oxygen the PDC is the only enzyme that catalyses the reaction from pyruvate to acetyl-CoA. Addition of lipoic acid to the medium resulted in an increase in $J_{\mathrm{PDC}}$ from 1.5 to $6.2 \mathrm{mmol}$ (g dry wt $)^{-1} h^{-1}$. Furthermore, as observed under anaerobic conditions, there was a marked decrease in acetoin production.

Growth of E. faecalis on SDM with pyruvate as energy source and without the addition of lipoic acid was only possible at pH 7.5 and higher (Table 3). When lipoic acid was added to the medium, the cells were able to grow at low $\mathrm{pH}$ values and $J_{\mathrm{PDC}}\left[9 \cdot 4 \mathrm{mmol}(\mathrm{g} \text { dry wt })^{-1} \mathrm{~h}^{-1}\right.$ at $\mathrm{pH} 6.0]$ was calculated. Apparently, an active PDC is required for growth on pyruvate at low $\mathrm{pH}$ values. This requirement will reside in the inability to produce ATP except via acetate formation, and the low PFL activities in pyruvate cultures at low $\mathrm{pH}$ values (Table 3 and Snoep et al., 1990).

The activities of the pyruvate-catabolizing enzymes were also measured under glucose excess (potassiumlimited) conditions. Under these conditions the fermentation of glucose was almost homolactic with only a minor portion of the pyruvate converted into acetyl-CoA by PFL (Table 4). The dry weight increased markedly with the culture $\mathrm{pH}$ value; at $\mathrm{pH} 6.0,7.0$ and 8.0 , values of $0.7,1.2$ and $1.8 \mathrm{~g} \mathrm{l}^{-1}$ were measured, respectively.

Clearly, the addition of lipoic acid had a large effect on the steady-state product formation of E. faecalis, when grown on SDM. In cell-free extracts of cells grown on SDM without added lipoic acid, no overall PDC activity could be measured, but activity of the dihydrolipoyl transacetylase (enzyme 2) and dihydrolipoyl dehydrogenase (enzyme 3) was observed (data not shown). These results indicate that the components of the PDC were present but could not be active due to the absence of lipoamide. To investigate the kinetics of reconstitution of the PDC and the effect of activation of the PDC on product formation, lipoic acid was added instantaneously ('pulse') to the culture vessel of a glucose + pyruvate-limited chemostat culture. Prior to the pulse, all acetate was formed via PFL, with a considerable rate of acetoin production $\left[q_{\text {acetoin }}=4 \cdot 9, q_{\text {acetate }}=4 \cdot 9, q_{\text {formate }}=\right.$ $5.0 \mathrm{mmol}(\mathrm{g} \text { dry wt })^{-1} \mathrm{~h}^{-1}$ ], whereas after the pulse, acetate $\left(\mathrm{CO}_{2}\right)$ and lactate were the sole products formed, and formate and acetoin washed out (Fig. 1). As in the 


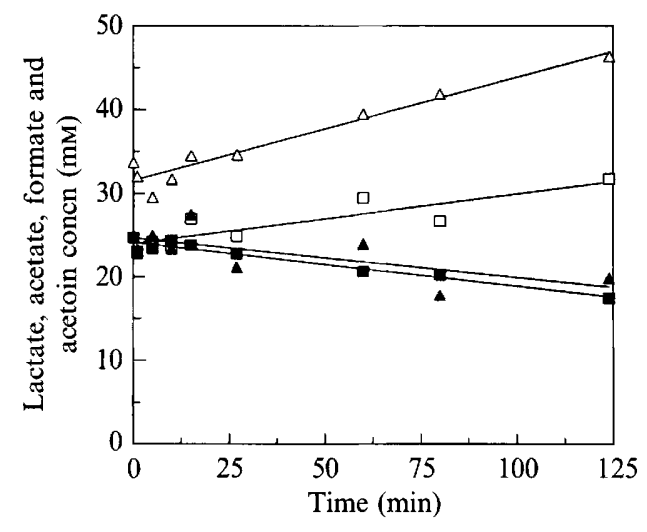

Fig. 1. Effect of a lipoic acid pulse ( $10 \mu \mathrm{M}$ final concentration) to a glucose + pyruvate-limited chemostat culture of $E$. faecalis $(D=$ $\left.0 \cdot 2 \mathrm{~h}^{-1}, \mathrm{pH}=6.0\right)$ on the production of lactate $(\triangle, 60 \mathrm{~mm}$ has been subtracted from the actual value), acetate $(\square)$, formate $(\boldsymbol{\Delta})$ and acetoin $(\boldsymbol{\square})$. Values are the average of three independent chemostat runs, $\mathrm{SD}<5 \%$ of the mean.



Fig. 2. Reconstitution of the PDC in vitro. Values are the average of two independent experiments, SD $<5 \%$ of the mean.

steady-state cultures, activation of the PDC had a profound effect on pyruvate catabolism [after the pulse the $J_{\mathrm{PDC}}$ was $8.1 \mathrm{mmol}\left(\mathrm{g}\right.$ dry wt) ${ }^{-1} \mathrm{~h}^{-1}$ ]. Since the effect was observed immediately after the addition of lipoic acid to the culture vessel, no large changes in enzyme concentration are likely to have occurred and the observed changes in the catabolic flux can be ascribed to regulation of enzyme activity rather than regulation of gene expression. Probably activation of the PDC resulted in a lowering of the internal pyruvate concentration to a level that is too low for the $\alpha$-ALS to be active (as described above).

Reconstitution of apo-PDC was also performed in vitro in cell-free extracts of cells grown on SDM (which lacks lipoic acid). The kinetics of the reconstitution were rather complex. After an initial pre-incubation of the assay mix in the presence of ATP and lipoic acid, the reaction was started by the addition of pyruvate, but there was a lag before the enzyme became active. The duration of this lag phase was dependent on the length of the pre-incubation time, the longer the incubation period the shorter the lag phase: after 20 min incubation, a lag phase of 6 min was observed, whereas after 95 min preincubation it was only $2 \mathrm{~min}$. That this lag phase was not observed by other investigators who worked on the reconstitution of apo-PDC might be due to their different assay methods, e.g. using propionyl phosphate formation after $30 \mathrm{~min}$ incubation (Reed et al., 1958) or electrophoresis (Brookfield et al., 1991). Fig. 2 shows that maximum activation of the apo-PDC occurred after about $90 \mathrm{~min}$. The origin of the discrepancy between the in vivo (immediate activation) and in vitro (slow activation) reconstitution is not understood.

\section{Discussion}

The inability to produce lipoic acid makes E. faecalis most suited to study the requirement for this vitamin and allows an investigation into the effects of activation of the PDC on pyruvate catabolism. Furthermore, the unique characteristic of this complex in being active under anaerobic conditions raises the question as to its physiological relevance.

From the potassium-limited cultures it is clear that under conditions of glucose excess no lipoic acid is needed for catabolic processes; almost all the pyruvate is reduced to lactate. However, when pyruvate had to be converted into products other than lactate to maintain redox neutrality (e.g. when the energy source was more oxidized than glucose), PFL and $\alpha$-ALS were active. Whenever lipoic acid was added to such a culture, a decrease in the activities of the PFL and $\alpha$-ALS was observed and the PDC became active. This effect was observed in steady state cultures and was instantaneous after addition of lipoic acid to the growing culture. As shown previously, $\alpha$-ALS, the first enzyme in the catabolic route to acetoin, has a very low affinity for pyruvate and can only be active when the intracellular pyruvate concentration is high (Snoep et al., 1992b). Most probably activation of the PDC by addition of lipoic acid to the medium resulted in a decrease in the internal pyruvate concentration to a value too low for $\alpha$-ALS to be active. This hypothesis is supported by the observation that relief of the glucose + pyruvate limitation by adding pyruvate to the culture vessel $(50 \mathrm{~mm}$ final concentration), resulted in a complete conversion of pyruvate into acetoin (data not shown).

An increase of the steady-state dry weight in potassium-limited cultures (with ammonium chloride as the nitrogen source) has been observed in Gram-negative as well as in Gram-positive organisms and in the yeast Candida utilis (Mulder, 1988; Buurman et al., 1989; Buurman, 1991; Hardy, 1992). The model that was 
proposed by Buurman et al. (1989) to explain these results should be valid for many microbial species. Indeed, our data show that for the Gram-positive $E$. faecalis this generalization is legitimate.

The experiments performed with the SDM without added lipoic acid and with pyruvate as energy source show that an active PDC is necessary for growth under these conditions. With energy sources other than pyruvate the physiological advantage of anaerobic activity of the PDC in vivo is less clear: during glucose catabolism the advantage of an anaerobic $J_{\mathrm{PDC}}$ cannot be to increase the flux to acetate, as each mole of pyruvate that is catabolized via the PDC will, as a consequence of the generation of additional NADH, lead to the formation of a mole of ethanol. If all glucose was converted via the PDC, the fermentation would be homo-ethanologenic and the efficiency (2 ATP per glucose) would be equal to a homolactic fermentation. Bacterial cells are not usually adapted to grow with maximal efficiency with respect to ATP generation (per substrate consumed, e.g. Van Dam et al., 1988), but strive for a maximal ATP production rate. However, it is not very likely that with energy sources other than pyruvate this rate can be increased by a PDC that is active under anaerobic conditions, since streptococci probably have an over-capacity for LDH (e.g. Snoep et al., 1992a). Most probably the PDC provides $E$. faecalis with a greater catabolic flexibility, enabling this organism to grow on a large number of energy sources under a wide range of conditions. In addition, it may be that PDC activity provides the cell with a means to circumvent excessive acid production (acetate plus formate) by forming acetate, ethanol and $\mathrm{CO}_{2}$ at low $\mathrm{pH}$. Regulation of the complex on the level of synthesis as well as on the level of activity ensures that it will not be active under conditions in which a high $\mathrm{NADH} / \mathrm{NAD}$ ratio exists (i.e. during growth under anaerobic conditions with an energy source with a high degree of reduction). Activity of the PDC under such conditions would be highly unfavourable due to its extra NADH generation (compared to PFL). The observed dependency of the distribution of the carbon flux over the PDC and PFL with the redox potential of the NAD/NADH couple supports this idea.

\section{References}

Brookfield, D. E., Green, J., Ali, S. T., Machado, R. S. \& Guest, J. R. (1991). Evidence for two protein lipoylation activities in Escherichia coli. FEBS Letters 295, 13-16.

BuUrman, E. T. (1991). The Effect of Cations on Microbial Metabolism and Growth Energetics. PhD thesis, University of Amsterdam, The Netherlands.

Buurman, E. T., Pennock, J., Tempest, D. W., Teixeira de Mattos,
M. J. \& NeIJSSEL, O. M. (1989). Replacement of potassium ions by ammonium ions in different organisms grown in potassium-limited chemostat culture. Archives of Microbiology 152, 58-63.

Evans, C. G. T., Herbert, D. \& Tempest, D. W. (1970). The continuous culture of microorganisms. 2. Construction of a chemostat. Methods in Microbiology 2, 277-327.

Gornall, A. G., Bardawill, C. J. \& David, M. A. (1949). Determination of serum proteins by means of the biuret reaction. Journal of Biological Chemistry 177, 751-766.

HaRDY, G. P. M. A. (1992). Dual Glucose Metabolism of Pseudomonas Species in Chemostat Culture. $\mathrm{PhD}$ thesis, University of Amsterdam, The Netherlands.

Herbert, D., Phipps, P. J. \& Strange, R. E. (1971). Chemical analysis of microbial cells. Methods in Microbiology 5B, 209-344.

MULDER, M. M. (1988). Energetic Aspects of Bacterial Growth: a Mosaic Nonequilibrium Thermodynamic Approach. $\mathrm{PhD}$ thesis, University of Amsterdam, The Netherlands.

Patel, M. S. \& Roche, T. E. (1990). Molecular biology and biochemistry of pyruvate dehydrogenase complexes. FASEB Journal 4 , 3224-3233.

Perham, R. N. (1991). Domains, motifs, and linkers in 2-oxo acid dehydrogenase multi-enzyme complexes: a paradigm in the design of a multifunctional protein. Biochemistry 30, 8501-8512.

Perham, R. N., Packman, L. C. \& Radford, S. E. (1987). 2-Oxo-acid dehydrogenase multi-enzyme complexes: in the beginning and halfway there. Biochemical Society Symposia 54, 67-81.

REED, L. J. (1974). Multienzyme complexes. Accounts of Chemical Research 7, 40-46.

ReED, L. J., Leach, F. R. \& KoIKe, M. (1958). Studies on a lipoic acid activating system. Journal of Biological Chemistry 232, 123-142.

Snoep, J. L., Teixeira de Mattos, M. J., Postma, P. W. \& Neijssel, O. M. (1990). Involvement of pyruvate dehydrogenase in product formation in pyruvate-limited anaerobic chemostat cultures of Enterococcus faecalis NCTC 775. Archives of Microbiology 154, $50-55$.

Snoep, J. L., Teixeira de Matros, M. J. \& Neisssel, O. M. (1991). Effect of the energy source on the NADH/NAD ratio and on pyruvate catabolism in anaerobic chemostat cultures of Enterococcus faecalis NCTC 775. FEMS Microbiology Letters 81, 63-66.

Snoep, J. L., Westphal, A. H., Benen, J. A. E., Teixeria de Mattos, M. J., NeIJSSEL, O. M. \& DE KoK, A. (1992a). Isolation and characterisation of the pyruvate dehydrogenase complex of anaerobically grown Enterococcus faecalis NCTC 775. European Journal of Biochemistry 203, 245-250.

Snoep, J. L., Teixeira de Mattos, M. J., Starrenburg, M. J. C. \& HugENHOLTZ, J. (1992b). Isolation, characterization and physiological role of the pyruvate dehydrogenase complex and $\alpha$ acetolactate synthase of Lactococcus lactis subsp. lactis var. diacetylactis. Journal of Bacteriology 174, 4838-4841.

Takahashi, S., AbBe, K. \& Yamada, T. (1982). Purification of pyruvate formate-lyase from Streptococcus mutans and its regulatory properties. Journal of Bacteriology 149, 1034-1040.

Van Dam, K., Mulder, M. M., Teixeira de Mattos, J. \& Westerhoff, H. V. (1988). A thermodynamic view of bacterial growth. In Physiological Models in Microbiology, I, pp. 25-48. Edited by M. J. Bazin \& J. I. Prosser. Boca Raton: CRC Press.

WiELAND, O. H. (1983). The mammalian pyruvate dehydrogenase complex: structure and regulation. Reviews of Physiology, Biochemistry and Pharmacology 96, 123-170.

Wittenderger, O. H. \& Angelo, N. (1970). Purification and properties of a fructose-1,6-bisphosphate-activated lactate dehydrogenase from Streptococcus faecalis. Journal of Bacteriology 101, 717-724.

WoLIN, M. J. (1964). Fructose-1,6-bisphosphate requirement of streptococcal lactate dehydrogenase. Science 146, 775-776.

Yamada, T. \& CARLsson, J. (1975). Regulation of lactate dehydrogenase and change of fermentation products in streptococci. Journal of Bacteriology 124, 55-61.

Yeaman, S. J. (1989). The 2-oxo acid dehydrogenase complexes: recent advances. Biochemical Journal 257, 625-632. 